\title{
Clinical analysis of percutaneous closure atrial septal defects guided by thoracic echocardiography (Report of 90 cases)
}

\author{
chunping $\operatorname{li}^{1}$ and Feng Huang ${ }^{1}$ \\ ${ }^{1}$ Fujian Medical University
}

September 24, 2021

\begin{abstract}
Objective :The purpose of this research is to explore the clinical application prospect of percutaneous closure atrial septal defects guided by thoracic echocardiography. Methods : Selected 90 inpatients who were pure atrial septal defects from sep 2014 to December 2019, Under local anesthesia via femoral vein puncture closure atrial septal defects guided by thoracic echocardiography, Real-time evaluatie plugging result.The patients underwent follow-up echocardiography at 3 days, 3 months, 6 months, 12 months after surgery.Results Intraoperative occlusion was not successful in 3 cases(The plug is not fixed firmly, so withdraw the plug), Occluder were successfully implanted in 87 patients, there were no serious complications such as valvular injury, pericardial effusion - occluder off,five patients had a little residual shunt in 3 days after surgery, residual shunt disapper after 3 months underwent follow-up echocardiography, the rest of the patients does not appear residual shunt.. Conclusion :Percutaneous closure atrial septal defects guided by thoracic echocardiography had Superiority such as simplicity of operator 、 shorter operator time 、 less-injury 、 safety - fast recovery,the surgery has a broad clinic prospects.
\end{abstract}

